\title{
Cesarean Skin Scar Characteristics For Preoperative Prediction of Intra-Abdominal Adhesions At Repeat Cesarean Delivery; a Prospective Cohort Study
}

\author{
Mohammed Khalaf, Fatma Abdel-Reheem, Ihab El-Nashar, Ahmed M Abbas* and Mansour \\ A Khalifa
}

Department of Obstetrics and Gynecology, Assiut University, Women Health Hospital, Assiut, Egypt

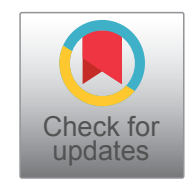

\begin{abstract}
Objective: To evaluate the association between cesarean skin scar characteristics and intra-abdominal adhesions at repeat cesarean delivery $(C D)$

Materials and methods: The current study was a prospective cohort study carried out in a tertiary Maternity hospital. Three hundred women with prior CD and scheduled for elective repeat CD were included. Preoperative evaluation of the cesarean skin scar characteristics was performed including scar appearance, pigmentation, length and width. During repeat $C D$, intra-abdominal adhesions were evaluated and classified according to the Nair's scoring system. The primary outcome was the rate and severity of adhesions and their association with the different skin scar characteristics.

Results: No difference between women with flat scars versus non-flat scars regarding the presence of adhesions $(p=$ $0.148)$. However, dense adhesions were significantly present in women with non-flat scars $(71.7 \%)$ versus $(46.1 \%)$ in the other group $(p=0.001)$. No difference between women with hyper pigmented and non-pigmented scars regarding the presence of adhesions $(p=0.448)$. Correlation analysis shows that Nair's score had a significant positive moderate correlation with scar length $(r=0.575, p=0.032)$, however no correlation with scar width $(r=0.137, p=0.082)$.
\end{abstract}

Conclusion: Cesarean skin scar length and presence of non-flat scars could be associated with the presence of intraabdominal adhesions during repeat $\mathrm{CD}$.

\section{Keywords}

Cesarean delivery, Cesarean scar, Pelvic adhesions, Nair's score

\section{Introduction}

Cesarean delivery $(C D)$ is one of the most commonly performed surgical operations worldwide. The rate of $C D$ is rising and thus increases the rate of intra-abdominal adhesions [1]. Intestinal injuries, bladder injuries, adhesive intestinal obstruction, chronic pelvic pain, and subsequent infertility could be serious complications of repeated abdominal surgery [2]. Even optimal surgical techniques cannot prevent adhesion formation completely.

There is no reliable way to predict the presence of intra-abdominal adhesions before performing $C D$ up till now. Several methods have been reported in the literature for prediction of the presence of adhesions after abdominal operations such as high-resolution ultrasonography [3], magnetic resonance imaging [4] and skin scar healing properties $[5,6]$. Anticipating adhesions is essential to prevent complications by ensuring that the required preoperative preparations are in place and/or that appropriate cases are referred to a tertiary center.
Skin incisions do not heal homogeneously. Since the abdominal skin scar of a previous CD and intra-abdominal adhesions are both tissue healing processes [7], in flat scars, the re-modeling and maturation of wound healing are assumed to be perfect. Apparently, the same applied to the internal (intra-abdominal) healing, with less scaring.

Therefore, the current study aims to evaluate the association between cesarean skin scar characteristics and intra-ab-

*Corresponding author: Ahmed M Abbas, Department of Obstetrics and Gynecology, Assiut University, Women Health Hospital, Assiut, Egypt

Accepted: September 26, 2020

Published online: September 28, 2020

Citation: Khalaf M, Abdel-Reheem F, El-Nashar I, et al. (2020) Cesarean Skin Scar Characteristics For Preoperative Prediction of Intra-Abdominal Adhesions At Repeat Cesarean Delivery; a Prospective Cohort Study. Annals Gynecol Obstet 4(1):72-76 
Citation: Khalaf M, Abdel-Reheem F, El-Nashar I, et al. (2020) Cesarean Skin Scar Characteristics For Preoperative Prediction of IntraAbdominal Adhesions At Repeat Cesarean Delivery; a Prospective Cohort Study. Annals Gynecol Obstet 4(1):72-76

dominal adhesions at repeat $C D$.

\section{Materials and Methods}

\section{Study type, settings, and duration}

A prospective cohort study was carried out in a tertiary Maternity hospital between November 2016 and March 2018. The study protocol was approved by the institutional ethical review board. After a discussion of the nature of the study, written consent was obtained from all study participants before enrollment.

\section{Study participants}

We invited all women scheduled for elective repeat $C D$ to participate in the study. We included pregnant women between 37 and 40 weeks gestation with at least previous one $\mathrm{CD}$. We excluded women with previous abdominopelvic surgery other than $C D$, midline incisions, post-operative wound infections, history of pelvic inflammatory disease, endometriosis, and chronic use of corticosteroids. Additionally, women scheduled for emergency $C D$ and those suspected to have morbidly adherent placenta were excluded from the study. Finally, women declined to participate in the study were excluded.

\section{Study tools \& Intervention}

At inclusion; age, parity, gestational age, previous miscarriage, and previous CD were evaluated. Body mass index (BMI) was calculated for every participant.

On the examination bed, the cesarean skin scar characteristics were evaluated regarding those items:

1. Abdominal scar length and width were measured with a plastic ruler in $\mathrm{cm}$,

2. The shape of the scar: Scars were categorized as elevated, flat or depressed relative to the level of the surrounding skin. Flat scar defined as a scar flushed with the skin and seen as a line not depressed or elevated. Depressed scar is seen as a dimpled scar or depressed below the skin surface. Elevated scar is seen above the level of the skin.

3. The color of the scar: Scars were categorized as hyper pigmented or non-pigmented compared to the neighboring skin.

During cesarean, intra-abdominal adhesions were evaluated and classified according to the Nair's scoring system [8] Nair et al. described their classification system as follows: Grade 0: Complete absence of adhesions, Grade 1: Single band of adhesion between viscera or from one viscus to the abdominal wall, Grade 2: Two bands either between viscera or from viscera to the abdominal wall, Grade 3: More than two bands between viscera or from viscera to the abdominal wall and Grade 4: Multiple dense adhesions or viscera directly adherent to the abdominal wall, irrespective of number or extent of adhesive bands.

A modified Nair's scoring system was used to evaluate the association between skin scar characteristics and the density of adhesions. According to this modified classification, adhe- sions were classified as filmy [grade 1 or 2] or dense [grade 3 or 4].

All CDs were performed by one of the study investigators. A detailed report was completed regarding the intra operative adhesions density and locations after the surgery. The primary outcome of the study was the difference in the rate of intra-abdominal adhesions according to the skin scar characteristics.

\section{Statistical analysis}

Data were collected, tabulated, then analyzed using IBM ${ }^{\odot}$ SPSS $^{\odot}$ Statistics version 22 (IBM ${ }^{\odot}$ Corp., Armonk, NY). Quantitative data were presented as mean \pm SD and compared using the unpaired t-test. Qualitative data were presented as number and percentage and compared using the Chi-square test. Correlation analysis was performed between cesarean scar length and width with Nair's score. Additionally, a multivariate logistic regression model was performed for predictors of intra-abdominal adhesions. P-value was considered significant if less than 0.05 .

\section{Results}

We enrolled 300 women in the study after exclusion of 46 cases as they did not meet the inclusion criteria. The mean gestational age of pregnancy was $38.26 \pm 1.13$ weeks. Eighty women $(26.7 \%)$ had a history of previous three CDs or more. Of 300 women enrolled in the study, $39(13 \%)$ had no adhesions, 17 (42.4\%) had filmy adhesions, and 29 (44.7\%) had dense adhesions.

Table 1 shows the results of analysis of clinical, skin scar characteristics and intra-abdominal adhesions in relation to scar appearance. No significant difference regarding BMI, number of previous CDs, cesarean skin scar length and width between both groups. Women with non-flat scar had a significantly higher frequency of hyper pigmented scars than women with flat ones ( $92.3 \%$ vs. $33.6 \%, p=0.000)$. No difference between both groups regarding the presence of intra-abdominal adhesions $(p=0.148)$. However, dense adhesions were significantly present in women with non-flat scar $(71.7 \%)$ versus $(46.1 \%)$ in the other group $(p=0.001)$.

The sensitivity of non-flat scars for prediction of adhesions was $20.3 \%$ ( $95 \% \mathrm{Cl}$ : 15.6 - 25.7), specificity $69.2 \%$ (95\% Cl: 52.4 - 83) and the accuracy was $26.7 \%$ (95\% Cl: 21.7 - 32). However, the sensitivity of non-flat scars for prediction of dense adhesions was $28.4 \%$ (95\% Cl: 20.9 - 36.8), specificity $88.2 \%$ (95\% Cl: $81.3-93.2)$ and the accuracy was $57.5 \%(95 \% \mathrm{Cl}$ : 51.2 - 63.5).

Table 2 shows the results of analysis of clinical, skin scar characteristics and intra-abdominal adhesions according to scar pigmentation. No significant difference regarding BMI, number of previous CDs, cesarean skin scar length and width between women with hyperpigmented and non-pigmented scars. Moreover, no difference between both groups regarding the presence of intra-abdominal adhesions $(p=0.448)$. The sensitivity of hyper-pigmented scars for prediction of adhesions was $46.4 \%$ ( $95 \% \mathrm{Cl}: 40.2$ - 52.6), specificity $53.8 \%$ (95\% Cl: 37.2 - 69.9) and the accuracy was $47.3 \%$ (95\% Cl: 41.6 - 53.1). 
Citation: Khalaf M, Abdel-Reheem F, El-Nashar I, et al. (2020) Cesarean Skin Scar Characteristics For Preoperative Prediction of IntraAbdominal Adhesions At Repeat Cesarean Delivery; a Prospective Cohort Study. Annals Gynecol Obstet 4(1):72-76

Table 1: Clinical, skin scar characteristics and intra-abdominal adhesions among study participants according to scar appearance.

\begin{tabular}{|l|l|l|l|}
\hline Characteristics & Flat scar $(\mathbf{n}=\mathbf{2 3 5})$ & Non-flat scar $(\mathbf{n}=\mathbf{6 5})$ & P-value \\
\hline BMI $\left(\mathrm{Kg} / \mathrm{m}^{2}\right)^{\#}$ & $25.65 \pm 3.23$ & $24.82 \pm 2.81$ & 0.060 \\
\hline Number of previous CD $\#$ & $2.14 \pm 1.16$ & $1.95 \pm 0.93$ & 0.246 \\
\hline Previous $\geq 3 \mathrm{CD}, n(\%)$ & $67(28.5)$ & $13(20.0)$ & 0.205 \\
\hline Scar length (cm) $)^{\#}$ & $14.45 \pm 1.75$ & $14.31 \pm 2.21$ & 0.583 \\
\hline Scar width (mm) & $2.38 \pm 1.11$ & $2.43 \pm 1.26$ & 0.414 \\
\hline Scar pigmentation, $n$ (\%) & & & $0.000^{*}$ \\
\hline Hyper pigmented & $79(33.6)$ & $60(92.3)$ & \\
\hline Non-pigmented & $156(66.4)$ & $5(7.7)$ & 0.148 \\
\hline Intra-abdominal adhesions, $n$ (\%) & & & \\
\hline Absent & $27(11.5)$ & $12(18.5)$ & \\
\hline Present & $208(88.5)$ & $53(81.5)$ & $0.001^{*}$ \\
\hline Grade 1 & $49(23.6)$ & $1(1.9)$ & \\
\hline Grade 2 & $63(30.3)$ & $14(26.4)$ & \\
\hline Grade 3 & $49(23.6)$ & $17(32.1)$ & \\
\hline Grade 4 & $47(22.5)$ & $21(39.6)$ & \\
\hline
\end{tabular}

CD: Cesarean Delivery; BMI: Body Mass Index

\#Data are presented as mean \pm standard deviation

*Statistical significant difference

Table 2: Clinical, skin scar characteristics and intra-abdominal adhesions among study participants according to scar pigmentation.

\begin{tabular}{|l|l|l|l|}
\hline Characteristics & Hyper-pigmented scar $(\mathbf{n = 1 3 9 )}$ & Non-pigmented scar $(\mathbf{n}=161)$ & P-value \\
\hline BMI $\left(\mathrm{Kg} / \mathrm{m}^{2}\right) \#$ & $25.73 \pm 3.56$ & $25.24 \pm 2.97$ & 0.174 \\
\hline Number of previous CD \# & $2.04 \pm 1.21$ & $2.14 \pm 1.04$ & 0.442 \\
\hline Previous $\geq 3 \mathrm{CD}, n(\%)$ & $32(23.0)$ & $48(29.8)$ & 0.193 \\
\hline Scar length $(\mathrm{cm})^{\#}$ & $14.35 \pm 2.06$ & $14.48 \pm 1.66$ & 0.566 \\
\hline Scar width $(\mathrm{mm}){ }^{\#}$ & $2.78 \pm 1.01$ & $2.63 \pm 1.16$ & 0.387 \\
\hline Intra-abdominal adhesions, $n(\%)$ & & & 0.448 \\
\hline Absent & $18(12.9)$ & $21(13.1)$ & \\
\hline Present & $121(87.1)$ & $140(86.9)$ & \\
\hline Grade 1 & $19(15.7)$ & $31(22.1)$ & \\
\hline Grade 2 & $35(28.9)$ & $42(30.0)$ & 0.521 \\
\hline Grade 3 & $30(24.8)$ & $36(25.7)$ & \\
\hline Grade 4 & $37(30.8$ & $31(22.2)$ & \\
\hline
\end{tabular}

CD: Cesarean Delivery; BMI: Body Mass Index

\# Data are presented as mean \pm standard deviation.

Table 3 shows that no difference between women with or without intra-abdominal adhesions regarding any of the skin scar characteristics or the number of previous CDs. However, women with dense adhesions were more commonly have non-flat scars with higher length than those with fine adhesions ( $p=0.003$ and 0.004 , respectively) while no difference regarding scar pigmentation $(p=0.321)$. Additionally, dense adhesions were more common in women with previous 3 or more CDs $(p=0.000)$.

Correlation analysis shows that Nair's score had a significant positive moderate correlation with scar length $(r=0.575$, $p=0.032)$, however no correlation with scar width $(r=0.137$, $p=0.082$ ). According to the results of multivariate regression analysis, non-flat scar and hyper-pigmented scar were not significant predictors for development of intra-abdominal adhesions $(\mathrm{OR}=2.11,95 \% \mathrm{Cl}: 0.93-4.81, \mathrm{P}=0.070$ and $\mathrm{OR}=$ $0.95,95 \% \mathrm{Cl}: 0.47-1.89, \mathrm{p}=0.88$, respectively).

\section{Discussion}

In the present study, we found that cesarean skin scar length and presence of non-flat scars could be associated with intra-abdominal adhesions during repeat CD. Intra-ab- 
Citation: Khalaf M, Abdel-Reheem F, El-Nashar I, et al. (2020) Cesarean Skin Scar Characteristics For Preoperative Prediction of IntraAbdominal Adhesions At Repeat Cesarean Delivery; a Prospective Cohort Study. Annals Gynecol Obstet 4(1):72-76

Table 3: Clinical, skin scar characteristics among study participants according to Nair's score

\begin{tabular}{|c|c|c|c|c|c|c|}
\hline Characteristics & $\begin{array}{l}\text { No Adhesions } \\
\text { (score } 0)(n=39)\end{array}$ & $\begin{array}{l}\text { Adhesions (score } \\
1-4)(n=261)\end{array}$ & $\begin{array}{l}\text { Filmy adhesions } \\
(n=127)\end{array}$ & $\begin{array}{l}\text { Dense adhesions } \\
(n=134)\end{array}$ & P-value $^{1}$ & P-value ${ }^{2}$ \\
\hline Previous $\geq 3 \mathrm{CD}, n$ (\%) & 7 (17.9) & 73 (28.0) & 16 (12.6) & $57(42.5)$ & 0.244 & $0.000^{*}$ \\
\hline Scar length $(\mathbf{c m})^{\#}$ & $14.69 \pm 1.95$ & $14.38 \pm 1.84$ & $14.04 \pm 1.76$ & $14.69 \pm 1.87$ & 0.327 & $0.004^{*}$ \\
\hline Scar width $(\mathrm{mm})^{\#}$ & $2.14 \pm 1.25$ & $2.29 \pm 1.36$ & $2.19 \pm 1.07$ & $2.31 \pm 1.12$ & 0.149 & 0.421 \\
\hline \multicolumn{7}{|l|}{ Scar appearance, $n(\%)$} \\
\hline Elevated & $12(30.8)$ & $49(18.8)$ & $15(11.8)$ & $34(25.4)$ & & \\
\hline Flat & $27(69.2)$ & $208(79.7)$ & $112(88.2)$ & 96 (71.6) & 0.176 & $0.003^{*}$ \\
\hline Depressed & 0 & $4(1.5)$ & 0 & $4(3.0)$ & & \\
\hline \multicolumn{7}{|c|}{ Scar pigmentation, $n$ (\%) } \\
\hline Hyper pigmented & $18(46.2)$ & $121(46.4)$ & $55(43.3)$ & $67(50.0)$ & 0.327 & 0.321 \\
\hline Non-pigmented & $21(53.8)$ & $140(53.6)$ & $72(56.7)$ & $67(50.0)$ & & \\
\hline
\end{tabular}

CD: Cesarean Delivery

\# Data are presented as mean \pm standard deviation

*Statistical significant difference

P-value ${ }^{1}$ for the difference between patients with and without adhesions.

P-value ${ }^{2}$ for the difference between patients with filmy and dense adhesions.

dominal adhesions development after $C D$ is one of the significant worries for obstetricians since they may influence the future reproductive of those women. Long term infertility, chronic pelvic pain, and occurrence of ectopic pregnancy are common squeal for intra-abdominal adhesions [9], besides the likelihood of traumatic injuries and blood loss during future adhesiolysis [10]. There is no gold standard method for prediction of intra-abdominal adhesions in spite of the previously reported extensive publications.

Pergialiotis et al. conducted a meta-analysis of all observational studies using cesarean skin scar characteristics for prediction of intra-abdominal adhesions [11]. They included five studies on 902 patients divided into two groups; patients with adhesions $(n=418)$ and without adhesions ( $n=484)$ [6,12-15]. In this meta-analysis, they reported a higher prevalence of intra-abdominal adhesions among patients with nonflat scars compared to those with flat scars [11]. The findings of our study are in disagreement with their results.

We found no difference between both groups regarding the presence of adhesions $(p=0.148)$. Moreover, they found depressed scars were predictive for the presence of adhesions (OR $=2.79,95 \% \mathrm{Cl}: 1.74-4.46, \mathrm{p}<0.0001)$, while elevated scars are not $(\mathrm{OR}=1.61,95 \% \mathrm{Cl}: 0.91-2.85, \mathrm{p}=0.10)$. In our study, we could not assess the predictive value of depressed scar due to the low number of patients $(n=4)$, however nonflat scars as a whole were not predictive for the presence of adhesions ( $\mathrm{OR}=2.11,95 \% \mathrm{Cl}: 0.93-4.81, \mathrm{P}=0.070)$.

The sensitivity of non-flat scar for prediction of adhesions was very poor according to Pergialiotis, et al.; $14 \%$ for depressed and $17 \%$ for elevated scars while the specificity was moderate (70\% for depressed and $74 \%$ for elevated scars) [11]. This was matched with our results in which the sensitivity of non-flat scars was $20.3 \%$, and the specificity was $69.2 \%$.

Pergialiotis et al. reported that scar pigmentation was also predictive of the presence of adhesions with no statistical significance $(\mathrm{OR}=1.68,95 \% \mathrm{Cl}: 0.86-3.26, \mathrm{p}=0.13)$ [11]. Our results disagree with them as hyper pigmented scars were not predictive for the presence of adhesions (OR $=0.95,95 \% \mathrm{Cl}$ : $0.47-1.89, \mathrm{p}=0.88)$. The sensitivity of hyper-pigmented scars in our study was $46.4 \%$, and the specificity was $53.8 \%$ while pergialiotis, et al. found $27 \%$ sensitivity and $60 \%$ specificity for prediction of adhesions.

Regarding the scar length and width, previous studies in the literature reported conflicting results; therefore we cannot depend on any of them for prediction of adhesions. Our study found no difference between women with or without adhesions regarding the scar length and width. However, we found a significant positive moderate correlation between only the scar length and severity of adhesions $(r=0.575, p$ $=0.032$ ).

Our results agreed with Kahyaoglu et al. who reported similar scar length in both groups $(p>0.005)$ [13]. On the opposite hand, they found that the scar width was larger in patients with adhesions $(p=0.003)$.

Additionally, Salim, et al. reported no difference in the scar length $(p=0.06)$ and width among both groups $(p=0.5)$ [12]. Our results were not matched with Khlifi, et al. who reported that the mean length of scars was higher in patients with adhesions $(p<0.001)$, while the width was similar $(p>$ 0.057) [14].

In our study, patients with non-flat scars are more likely to have dense adhesions (71.7\%) compared to those with flat scars $(46.1 \%)(p=0.001)$. This coincides with Dogan et al. who observed that the rate of dense adhesions was low (6.8\%) in women with flat scars versus $12.7 \%$ and $31.4 \%$ in women with depressed and elevated scars respectively [6]. Similarly, Sham et al. reported that $70.4 \%$ of women with non-flat scars had dense adhesions versus $29.6 \%$ women with flat scars ( $p$ 
Citation: Khalaf M, Abdel-Reheem F, El-Nashar I, et al. (2020) Cesarean Skin Scar Characteristics For Preoperative Prediction of IntraAbdominal Adhesions At Repeat Cesarean Delivery; a Prospective Cohort Study. Annals Gynecol Obstet 4(1):72-76

$=0.000$ ) [15]. On the contrary, Salim et al. and Kahyaoglu et al. did not find any difference between dense and filmy adhesions among women with different scar appearance [12,13].

In our study, the presence of non-flat scars was $88.2 \%$ specific for prediction of dense adhesions. This means that $88.2 \%$ of women that have neither elevated nor depressed scars will have no dense adhesions during repeat $\mathrm{CD}$. However, the sensitivity was too low (28.4\%); this means that only $28.4 \%$ of women that have non-flat scars will have dense adhesions during repeat CS. Therefore, the scar appearance seems to be a good negative test for prediction of dense adhesions.

When patients with dense adhesions were compared with those with filmy adhesions, a statistically significant difference was found regarding the percentage of previous $\geq 3 C D$, scar appearance and length, while no difference regarding scar pigmentation and width $(p>0.05)$. This was not coinciding with Kahyaoglu et al. results which found no difference between both groups regarding all mentioned variables [13].

The strengths of the current study are the large sample size of the study participants. Additionally, the assessment of cesarean skin scar characteristics is non-invasive, simple, a cheap tool with no need for special training; therefore it can be performed by medical interns or nurses. Further trials to confirm our findings are recommended.

The limitations of the current study included the lack of operative reports of the previous CDs as the presence of any extension of uterine incision, closure or non-closure of the visceral/parietal peritoneum, and the type of suture material used. All these factors might affect the presence of adhesions and the predictive value of skin scar characteristics.

\section{Conclusions}

In conclusion, cesarean skin scar length and presence of non-flat scars could be associated with the presence of intra-abdominal adhesions during repeat $C D$. This might influence the decision of who and where future repeat $C D$ could be done. We recommend inclusion of cesarean skin scar characteristics evaluation in the pre-operative examination for women with previous CD. Further trials should be instituted for evaluation of skin scar characteristics before any future laparoscopic procedures in patients with previous surgeries as it may affect the surgical approach choice.

\section{Funding}

The authors received no funding from an external source.

\section{Conflict of Interest}

The authors declare that they have no conflict of interest.

\section{References}

1. Tulandi T, Agdi M, Zarei A, et al. (2009) Adhesion development and morbidity after repeat cesarean delivery. Am J Obstet Gynecol 201: 56.e1-e6.

2. Nisenblat V, Barak S, Griness OB, et al. (2006) Maternal complications associated with multiple cesarean deliveries. Obstet Gynecol 108: 21-26.

3. Piccolboni D, Ciccone F, Settembre A (2009) High resolution ultrasound for pre-operative detection of intraperitoneal adhesions: An invaluable diagnostic tool for the general and laparoscopic surgeon. J Ultrasound 12: 148-150.

4. Zinther NB, Zeuten A, Marinovskij E, et al. (2010) Detection of abdominal wall adhesions using visceral slide. Surg Endosc 24: 3161-3166.

5. Tulandi T, Al-Sannan B, Akbar G, et al. (2011) Prospective study of intraabdominal adhesions among women of different races with or without keloids. Am J Obstet Gynecol 204: 132.e1-e4.

6. Dogan NU, Haktankacmaz SA, Dogan S, et al. (2011) A reliable way to predict intraabdominal adhesions at repeat cesarean delivery: Scar characteristics. Acta Obstet Gynecol Scand 90: 531-534.

7. Frishmann GN, Schwartz T, Hogan JW (2007) Caesarean section on the rise. Lancet 356: 1697.

8. Nair SK, Bhat IK, Aurora AL (1974) Role of proteolytic enzyme in the prevention of postoperative intraperitoneal adhesions. Arch Surg 108: 849-853.

9. Murphy DJ, Stirrat GM, Heron J (2002) ALSPAC Study Team. The relationship between caesaean section and sub fertility in a population-based sample of 14541 pregnancies. Hum Reprod 17: 1914-1917.

10. Ten Broek RP, Strik C, Issa Y, et al. (2013) Adhesiolysis-related morbidity in abdominal surgery. Ann Surg 258: 98-106.

11. Pergialiotis V, Frountzas M, Siotos C, et al. (2017) Cesarean wound scar characteristics for the prediction of pelvic adhesions: A meta-analysis of observational studies. J Matern Fetal Neonatal Med 30: 486-491.

12. Salim RY, Kadan Z, Nachum, et al. (2008) Abdominal scar characteristics as a predictor of intra-abdominal adhesions at repeat cesarean delivery. Fertil Steril 90: 2324-2327.

13. Kahyaoglu I, F Kayikcioglu, T Kinay, et al. (2014) Abdominal scar characteristics: Do they predict intra-abdominal adhesions with repeat cesarean deliveries? J Obstet Gynaecol Res 40: 1643-1648.

14. Khlifi A, Meddeb S, Kouira M, et al. (2015) Post-cesarean parietal scar characteristics are predictive of pelvic adhesions. A prospective cohort study. J Gynecol Obstet Biol Reprod (Paris) 44: 621-631.

15. Sham AA, Bahar A, AL-shahrani ME, et al. (2015) Cesarean section scar characteristics as a preoperative predictor of intra-abdominal adhesions. Med J Cairo Univ 83: 167-171.

DOI: $10.36959 / 468 / 469$

Copyright: () 2020 Khalaf M, et al. This is an open-access article distributed under the terms of the Creative Commons Attribution License, which permits unrestricted use, distribution, and reproduction in any medium, provided the original author and source are credited. 